\title{
Cannibalism and Infectious Disease: Friends or Foes?
}

\author{
Benjamin G. Van Allen, ${ }^{1,2, \star}$ Forrest P. Dillemuth, ${ }^{1}$ Andrew J. Flick, ${ }^{1}$ Matthew J. Faldyn, ${ }^{1}$ \\ David R. Clark, ${ }^{1,3}$ Volker H. W. Rudolf, ${ }^{4}$ and Bret D. Elderd ${ }^{1}$
}

1. Department of Biological Sciences, Louisiana State University, Baton Rouge, Louisiana 70803; 2. Marine Biology Research Division, University of California, San Diego, California 92093; 3. Department of Biology, University of Puerto Rico-Rio Piedras Campus, San Juan, Puerto Rico 00931; 4. Department of BioSciences MS-170, 6100 Main Street, Rice University, Houston, Texas 77005

Submitted December 14, 2016; Accepted April 12, 2017; Electronically published July 14, 2017

\begin{abstract}
Cannibalism occurs in a majority of both carnivorous and noncarnivorous animal taxa from invertebrates to mammals. Similarly, infectious parasites are ubiquitous in nature. Thus, interactions between cannibalism and disease occur regularly. While some adaptive benefits of cannibalism are clear, the prevailing view is that the risk of parasite transmission due to cannibalism would increase disease spread and, thus, limit the evolutionary extent of cannibalism throughout the animal kingdom. In contrast, surprisingly little attention has been paid to the other half of the interaction between cannibalism and disease, that is, how cannibalism affects parasites. Here we examine the interaction between cannibalism and parasites and show how advances across independent lines of research suggest that cannibalism can also reduce the prevalence of parasites and, thus, infection risk for cannibals. Cannibalism does this by both directly killing parasites in infected victims and by reducing the number of susceptible hosts, often enhanced by the stage-structured nature of cannibalism and infection. While the well-established view that disease should limit cannibalism has held sway, we present theory and examples from a synthesis of the literature showing how cannibalism may also limit disease and highlight key areas where conceptual and empirical work is needed to resolve this debate.
\end{abstract}

Keywords: cannibalism, transmission, parasite, disease, trophic transmission, epidemic.

\section{Introduction}

Cannibalism, the consumption of conspecifics, is widespread among animal species (Fox 1975; Polis 1981; Smith and Reay 1991; Ibáñez and Keyl 2010; Richardson et al. 2010). Understanding why cannibalism arises and how it is maintained in animal populations has long fascinated and challenged researchers (Fox 1975; Polis 1981; Elgar and Crespi 1992). Theory predicts that cannibalistic behavior should be determined by a balance between its costs (e.g., risk of injury

\footnotetext{
* Corresponding author; e-mail: bgvanallen@ucsd.edu.

ORCIDs: Van Allen, http://orcid.org/0000-0001-8489-4790; Clark, http:// orcid.org/0000-0003-3125-0160.

Am. Nat. 2017. Vol. 190, pp. 299-312. (C) 2017 by The University of Chicago. 0003-0147/2017/19003-57445\$15.00. All rights reserved.

DOI: $10.1086 / 692734$
}

or loss of inclusive fitness when harming kin) and benefits (e.g., gains in nutrition, reduction in competition; Polis 1981; Stenseth 1985; Elgar and Crespi 1992; Pfennig 1997; Richardson et al. 2010; Rudolf et al. 2010). The risk of infection is widely expected to select against cannibalism as well, since individuals are more likely to contract parasites when consuming conspecific versus heterospecific victims, which imposes a significant cost on cannibalistic behavior (Pfennig 2000). Yet evidence for disease imposing strong or widespread selection against cannibalistic behavior is limited and equivocal, despite the ubiquity of parasites and cannibalistic taxa (Reed et al. 1996; Rudolf and Antonovics 2007; Bolker et al. 2008; Sadeh et al. 2016). In this review and synthesis, we argue that it is important to consider the ecological and evolutionary effects of cannibalism on the parasite and its subsequent impact on parasite transmission. While this is rarely done, existing evidence suggests that when we consider the feedback between parasites and cannibalism, cannibalism may be more likely to reduce parasite prevalence and thus limit the negative effects of parasites on cannibalistic behavior.

The argument that the risks of parasite transmission through cannibalism outweigh any benefits arises from two central tenets. First, scientists were under the erroneous impression that incidences of cannibalism were extremely rare in nature (Eibl-Eibelsfelt 1961; Dawkins 1976). Although later work indicates that cannibalism occurs with high frequency across many taxa (Fox 1975; Polis 1981; Ibáñez and Keyl 2010; Richardson et al. 2010; Ohlberger et al. 2012), several studies suggested that the risk of parasite transmission could be a dominating factor limiting cannibalistic behavior and leading to its supposed infrequency across most species (Elgar and Crespi 1992; Pfennig et al. 1998; Curtis 2014). Second, studies of kuru in the Fore people of New Guinea, as well as other work with prion diseases, have indirectly promoted the idea that cannibalism could be a common mechanism responsible for the spread of parasites (Lindenbaum 1979; Klitzman et al. 1984; Collinge et al. 2006). Indeed, there is clear empirical evidence that some wildlife diseases can be spread through cannibalism (Pfennig et al. 1991; Boots 1998; Forbes 
2000; Pfennig 2000; Williams and Hernández 2006; Rudolf and Antonovics 2007; Bolker et al. 2008). As a result, the idea that cannibalism spreads disease or at least creates significant risk of infection for cannibals has continued to dominate the ecological literature.

In contrast, the ecological and evolutionary literature has paid surprisingly little attention to how cannibalism affects parasites and how this alters the relationship between cannibalism and disease. In this review, we take a comprehensive look at the interaction of cannibalism and parasites and synthesize the findings of recent theoretical and empirical studies. Based on this body of work, we identify and discuss three main mechanisms/concepts (fig. 1) whereby cannibalism can alter the prevalence and transmission of parasites (i.e., micro and, to a lesser extent, macroparasite pathogens). First, we discuss per capita effects of cannibalism on disease transmission (fig. 1A; Rudolf and Antonovics 2007). Second, we show how cannibalism can reduce the spread of diseases and does not always lead to higher risk of transmission for the cannibal (fig. 1B; Hart 1990; Reed et al. 1996; Boots 1998; Meunier 2015). Third, we discuss the ways that pathogens and cannibalism interact with stage-structured host populations and how this affects population and disease dynamics (fig. 1C; Claessen et al. 2004; Rudolf 2008; Ohlberger et al. 2012). Together, these mechanisms, which operate across taxa, alter multiple facets of parasite transmission and often make cannibalism more likely to prevent disease rather than disease limiting cannibalism. Following these sections, we discuss how details in the strategies of cannibals and hosts can alter both cannibal risk and parasite spread. We then consider the relationship between cannibalism and broader behavioral and evolutionary questions such as filial cannibalism. Finally, we highlight gaps in cur- rent research and suggest fruitful avenues for future empirical and theoretical research.

\section{Cannibalism as the Sole Form of Disease Transmission}

Cannibalism itself is unlikely to spread disease because the net number of infected individuals in the population cannot increase after one infected individual is consumed by a single cannibal (Rudolf and Antonovics 2007). Further, infected cannibals can only reduce the prevalence of the disease by continuing to eat infected and uninfected conspecifics (Rudolf and Antonovics 2007). This is shown in a susceptible-infected model with disease transmission occurring only through cannibalism (Rudolf and Antonovics 2007), where

$$
\begin{aligned}
& \frac{d S}{d t}=b N+e a N^{2}-a N S-\delta a g I S-d S, \\
& \frac{d I}{d t}=\delta a g I S-a N I-(d+v) I .
\end{aligned}
$$

Here the rate of change of susceptibles $(S)$ and infecteds $(I)$ is determined by the birth of new susceptibles $(b N$, where $N=S+I$ ); the increase in births due to resources gained from cannibals consuming victims, $e$; the cannibalism rate, $a$; the transmission of disease from infected victims to the cannibal(s), $\delta$; the group size of cannibals per victim, $g$; the background death rate, $d$; and the increase in infected individual mortality rate, $v$. For simplicity, this model assumes that infection takes hold immediately but only results in a higher mortality rate and does not alter cannibalistic behavior or production of new susceptible offspring. While cannibalistic transmission of disease can make cannibalism of in-

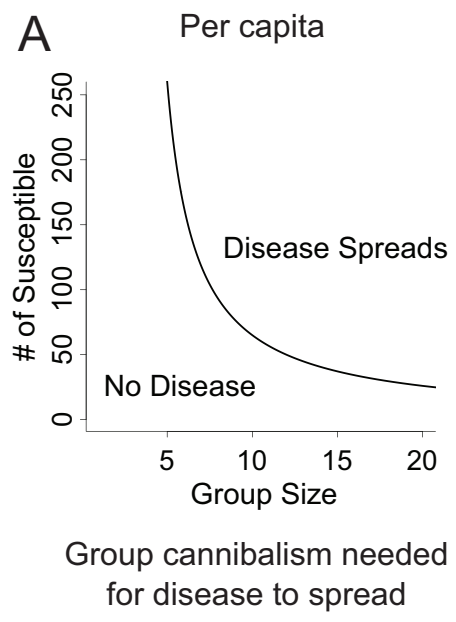

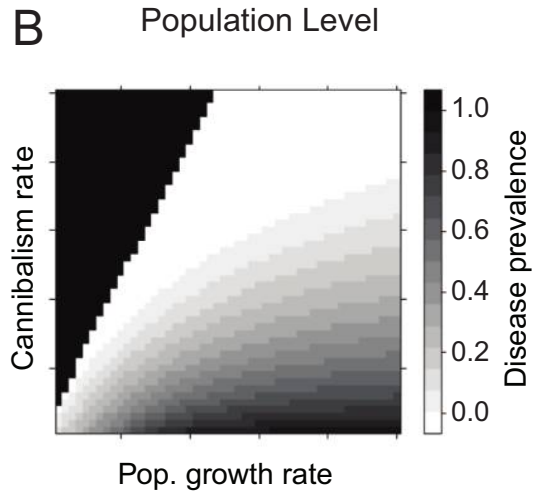

Decrease population below epizootic threshold

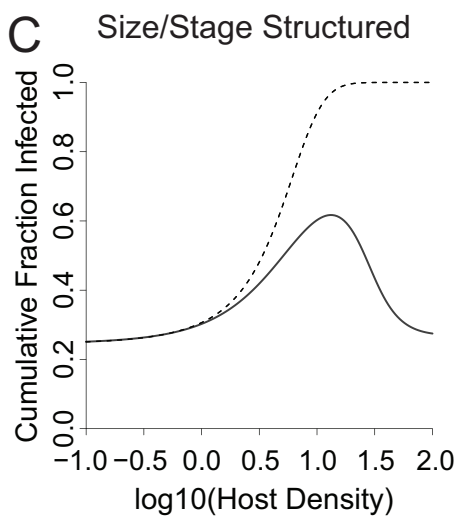

Removal of smaller infecteds, who contain less of a viral load

Figure 1: Three major mechanisms by which cannibalism negatively affects disease transmission: per capita $(A)$, population level $(B$; dark region in the upper left denotes areas of population extinction), and size/stage structured ( $C$; dashed line $=$ noncannibalistic population, solid line $=$ cannibalistic population). Per capita figure derived from Rudolf and Antonovics (2007). 
fected victims individually risky, this model indicates that a parasite spread only through one-on-one cannibalism cannot invade a cannibalistic population (Rudolf and Antonovics 2007). The findings of this analytical solution are borne out in the scarcity of diseases spread exclusively through cannibalism. While many diseases are spread somewhat incidentally though cannibalism (i.e., cannibalism is not a major transmission source), none is known to transmit solely through the killing and consumption of conspecifics, even for the few diseases where cannibalism represents a major course of transmission (Rudolf and Antonovics 2007). We caution that this pattern may partly be caused by the interaction between large populations and their diseases as compared to small populations. If cannibalistic transmission is perfect or near so, then even with only one-on-one cannibalism, population size goes down while the number of infections stays the same. This could lead to a noticeable increase in prevalence within a small cannibalistic population as cannibals pass the infection between increasingly fewer individuals. Even in this case, however, the actual number of infections will not increase due to cannibalism.

While one-on-one cannibalism cannot promote the spread of disease, cannibalism can still spread disease through a population by itself if, on average, more than one susceptible individual becomes infected per cannibalistic event (Rudolf and Antonovics 2007). For this to occur, however, multiple cannibals must consume the same infected victim. Even in the case of group cannibalism, transmission must also be high enough in concert with the average group size to maintain more than one new infection per cannibalistic event on infected hosts (Rudolf and Antonovics 2007). These rules were likely met in the case of the prion disease kuru, which spread in human populations in New Guinea, since multiple individuals contacted and consumed the remains of recently dead infected individuals, and the transmission rate was high (Lindenbaum 1979; Klitzman et al. 1984; Collinge et al. 2006). The spread of kuru through cannibalism alone is perhaps incidental to the situation, since prion proteins can spread through a number of mechanisms involving transfer of contaminated body fluids (Collinge 2001). In general, most examples of group consumption of conspecific victims come from humans and their closest relatives, the chimpanzees (Arcadi and Wrangham 1999; Mitani et al. 2002; Wilson et al. 2004; Rudolf and Antonovics 2007). Other sociable and predatory animals, such as wolves and lions, rarely consume conspecifics in groups (Rausch 1967; Bertram 1975). The prevalence of group cannibalism in invertebrates is less well known, but group cannibalism could occur in some species that frequently occur at high density or live in social groups (see, e.g., Wissinger et al. 2003; Vijendravarma et al. 2013). Given that cannibals rarely share infected victims in nature, theory suggests that lethal cannibalism is an insufficient transmission mechanism in the absence of any other transmission mech- anisms to ensure the persistence of parasites across most populations.

The absence of diseases spread exclusively through cannibalism is striking, given how common trophic transmission of parasites is in other systems. For example, the consumption of living victims is the only mode of transmission for many parasites, which spread from intermediate hosts (i.e., prey) to definite hosts (i.e., predators; Kuris 1974; Lafferty 1999; Johnson et al. 2010). Other forms of trophic transmission often considered cannibalism, such as nonlethal partial consumption (i.e., Sarcocystis in lizards [Matuschka and Bannert 1989] or devil facial tumor disease in Tasmanian devils [McCallum et al. 2009]), are not constrained in the same way as the killing and consumption of conspecifics (Rudolf and Antonovics 2007). Since infected individuals are not killed or removed from the populations in these systems, these transmission routes are more similar to standard frequency-dependent transmission, which allows transmission of the parasite to more than one conspecific at more than one time. Together these empirical and theoretical studies indicate that, compared to other forms of trophic transmission, cannibalism appears to be a disadvantageous mechanism for a pathogen to solely exploit without relying on other means of transmission.

\section{Cannibalism Culls the Infected and Reduces the Susceptible Population}

Not all diseases that can spread in cannibalistic populations are transmitted through cannibalism, yet cannibalism can still inhibit the spread of these diseases via two related mechanisms. First, cannibalism can be an intense form of density-dependent population regulation that decreases the number of susceptible hosts (Fox 1975; Polis 1981; Wagner and Wise 1996; Moksnes 2004; Bolker et al. 2008; Van Allen and Rudolf 2013; Sadeh et al. 2016). Since parasites transmitted via density-dependent means, as compared to frequency-dependent transmission, require a threshold density of susceptible hosts to invade a population (McCallum et al. 2001; Potapov et al. 2012), cannibalism could keep populations below this threshold. Thus, cannibalism can prevent or limit parasite invasion (Anderson and May 1980; McCallum et al. 2001; Potapov et al. 2012). Second, the consumption of infected conspecifics will not increase the number of infections (even if the parasite can be transmitted through cannibalism; see eq. [1]) and can decrease the number of infected individuals, reducing disease transmission even without density-dependent transmission (Reed et al. 1996; Boots 1998; Rudolf and Antonovics 2007; Bolker et al. 2008; Sadeh et al. 2016). Through these mechanisms, cannibalism is somewhat analogous to the culling of a wild animal population to prevent the spread of disease (Potapov et al. 2012; Brooks-Pollock et al. 2014). We can contrast the 
effects of cannibalism and proportional increases in mortality (such as culling) on the dynamics of a horizontally spread parasite using the general model,

$$
\begin{aligned}
\frac{d S}{d t}= & b N\left(1-\frac{N}{K}\right)-(m+h) S-\beta S I+e a N^{2} \\
& -a N S-\delta a I S, \\
\frac{d I}{d t}= & \beta S I-(m+\mu+h) I-a N I+\delta a I S,
\end{aligned}
$$

where $S$ and $I$ are the susceptible and infected individuals, respectively, and $N=S+I$. Susceptible individuals are added to the population assuming logistic growth $b(1-N / K)(N)$, die naturally at the rate of $m S$, and become infected through density-dependent horizontal (from individual to individual) transmission at rate $\beta$. Infected individuals are instantaneously infectious and have mortality increased by rate $\mu$. Both groups are equally susceptible to harvesting or culling, $h$, and are cannibalistic, without regard to infection status, at rate $a$. The parameters $e$ and $\delta$ represent the increase in reproductive output individuals receive from cannibalism per capita and the transmission rate of the disease through cannibalism on infected individuals, respectively.

Analysis of the model indicates that even low levels of cannibalism reduce the prevalence of infection in the population and can result in the exclusion of the pathogen across a wide range of parameter space (fig. $2 \mathrm{~A}$ ). Culling or harvesting, on the other hand, must be balanced very closely to the growth rate of the population, since only a narrow range of parameter values exist where prevalence is reduced (fig. 2B). Excluding the pathogen with culling requires especially finely tuned efforts, since only marginally higher rates of mortality will cause the population itself to collapse toward extinction. If the growth rate of the population is too high (greater than $h+m$, both of which are necessarily less than 1 ), then culling cannot exclude disease from the population (fig. 2B). This limitation is not nearly as strict with cannibalism, since mortality is limited by the population size itself, leading to a nonlinear relationship between population growth rate and pathogen exclusion (fig. $2 A$ ). Even in the simulation shown in figure $2 A$, where cannibalistic transmission occurs in more than half of cases $(d=0.6)$ and horizontal transmission is high $(\beta=0.5)$, there is substantial parameter space where the parasite either cannot invade or persists at low prevalence. The ability for cannibalism to reduce parasite spread and frequency in a population increases (all else equal) as transmission and population growth rates become lower (e.g., $\beta$, $\delta, e$, and/or $b$ decrease) but remains even when all cannibalistic events lead to a transmission event. From this epidemiological perspective, cannibalism has a strong tendency to reduce the prevalence of horizontally transmitted parasites, because it reduces population density and removes infected individuals (when $\delta<1$ and when infected individuals consume other infected individuals).

While this result indicates that cannibalism tends to reduce parasite prevalence in a population, cannibals still often face a higher risk of infection than noncannibals because they consume infected victims. The strength of this
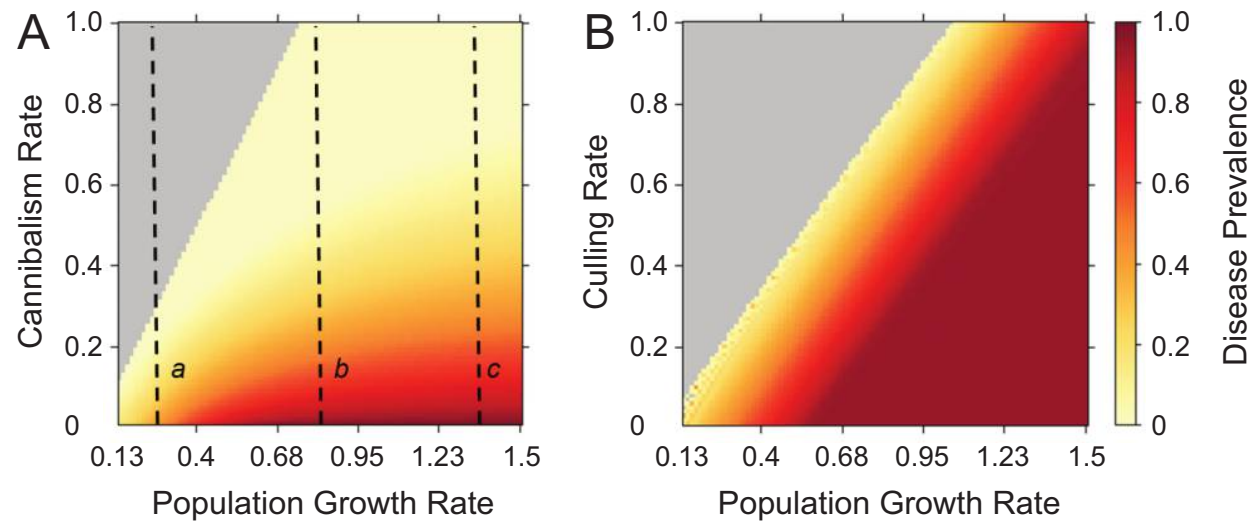

Figure 2: Effect of cannibalism $(A)$ and culling $(B)$ on equilibrium disease prevalence in a population across different population growth rates and cannibalistic or culling mortality rates using equation (2). Darker shading indicates a higher equilibrium prevalence of the disease within the population, and light gray areas in the upper left of both plots denote population extinction (equilibrium $(S+I)<1$ ). Light areas indicate a failure of the pathogen to invade and establish (indicating that the reproductive rate of the parasite, $R_{0}$, was $<1$ leading to equilibrium disease prevalence $<0.001$ ). In both plots, the parameter associated with increased mortality varies between 0 and 1 , and population growth rate varies between 0.13 and 1.5. Both ranges were explored with 100 equally spaced steps of parameter values (in all, 100,000 combinations) run for 100 time steps. The parameters used for cannibalism $(A)$ are $K=10,000, e=0.3, \delta=0.6, m=0.06, \beta=0.5$, and $\mu=0.5$, while for culling $(B), e=0$ and $\delta=0$; all other parameters are the same as in panel $A$. Each simulation began with $S=20$ and $I=0.5$ to simulate the invasion of a population by the parasite. Dashed lines through $A$ are used for figure 3 . 
infection risk varies with the ability of the parasites to transmit through cannibalism and the frequency of consuming infected individuals. Cannibalistic transmission $(\delta)$ is fixed in our population model (eq. [2]), but the frequency of consuming infected individuals changes with parasite prevalence for a given cannibalism rate and population size. Higher cannibalism rates reduce parasite prevalence and population size but increase the number of potentially infected victims that cannibals encounter. As a result, the per capita risk of infection through cannibalism changes nonlinearly (in eq. [2]) as cannibalism rates increase (fig. 3). At equilibrium densities, per capita infection risk for cannibals increases briefly as cannibalism rates increase, but this risk soon declines as higher cannibalism rates more effectively reduce parasite prevalence and the number of potential victims (fig. 3). As a result, infection risk for cannibals decreases as cannibalism rates become higher (relative to lower cannibalism rates) and eventually reaches zero when cannibalism prevents a parasite from spreading in the population (fig. 3). This illustrates that while cannibalism does increase individual risk of infection, more cannibalism may not result in increasing risk, and indeed the opposite can be true (Bolker et al. 2008). Furthermore, while cannibalism can be costly because of this increased infection risk, cannibalism has many other benefits that could overcome its costs (Fox 1975; Polis 1981; Pfennig 2000). More studies are needed that examine the eco-evolutionary costs and benefits of cannibalism in light of this nonlinear risk of infection to determine when parasites could select against cannibalism, and vice versa.

\section{Size- and Stage-Structured Cannibalism}

Who is a cannibal and who is a victim is frequently decided by variation in size or stage (Fox 1975; Polis 1981; Claessen et al. 2004; Ibáñez and Keyl 2010; Richardson et al. 2010; Miller and Rudolf 2011). Earlier life stages tend to be smaller and less able to defend themselves. Consequently, older, larger individuals typically consume earlier, smaller stages. Many parasites affect the size/stage structure of a population at the individual level, by altering or stopping growth and changing host behavior (Sait et al. 1994; Park et al. 1996; Kelehear et al. 2009; Hatcher et al. 2014; Bunke et al. 2015). Furthermore, larger and more mature individuals tend to be more resistant and require higher doses to become infected, making younger/smaller stages an easier target for parasites (Feng et al. 1985; Briggs and Godfray 1995; Boots 1998; Boff et al. 2000; Kelehear et al. 2009; Haislip et al. 2011; Harrison and Hoover 2012). Thus, increased parasite prevalence in a population also frequently increases size and activity differences among individuals and thereby increases the opportunity for targeted cannibalism of smaller infected conspecifics. Such size-specific effects of parasites can thus strongly interact with the population-culling effects of cannibalism by increasing both the reduction of host density and targeted removal of infected individuals, but potentially decreasing the consumption of infecteds by infecteds and increasing the quality of potential hosts when parasites spread through cannibalism. With this increased biological realism, the answers begin to lie in the details.

A particularly striking case of the interactions between cannibalism and parasite-induced variation in size/stage occurs in lepidopteran larvae infected with lethal baculoviruses. Baculoviruses are a large group of viruses that infect a number of lepidopteran species. Lepidopteran larvae inadvertently ingest viral particles in the environment, and the virus prevents the infected host from continuing its development, but healthy individuals continue to grow (Elderd 2013). This stunting effect can lead to substantial differences in size and stage between healthy and infected individuals (fig. 4; Elderd et al. 2008; Elderd and Reilly 2014). Despite being generally herbivorous, a high proportion of lepidopteran larvae are also cannibalistic (Richardson et al. 2010).
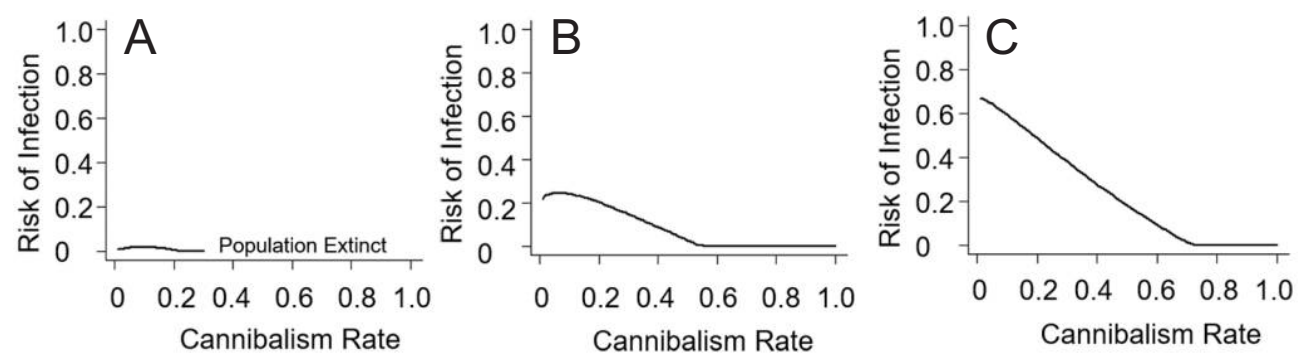

Figure 3: Risk of infection through cannibalism after the system reached equilibrium at the end of each simulation using equation (2). Each figure represents a slice through panel $A$ of figure 2. Instantaneous infection risk through cannibalistic activity (beyond any risk of infection through horizontal transmission) occurs when consuming conspecifics who may or may not be infected with a parasite that can transfer through cannibalism, but this risk does not linearly increase with increased cannibalistic activity. Infection risk is calculated by multiplying the cannibalism rate by the cannibalistic transmission rate $(\delta)$ and the number of infected individuals in the population at equilibrium (i.e., the number of infected potential victims). As a result, at equilibrium, infection risk $=a \times \delta \times I$. The cannibalistic transmission rate used here is as in figure $2, \delta=0.6$. 


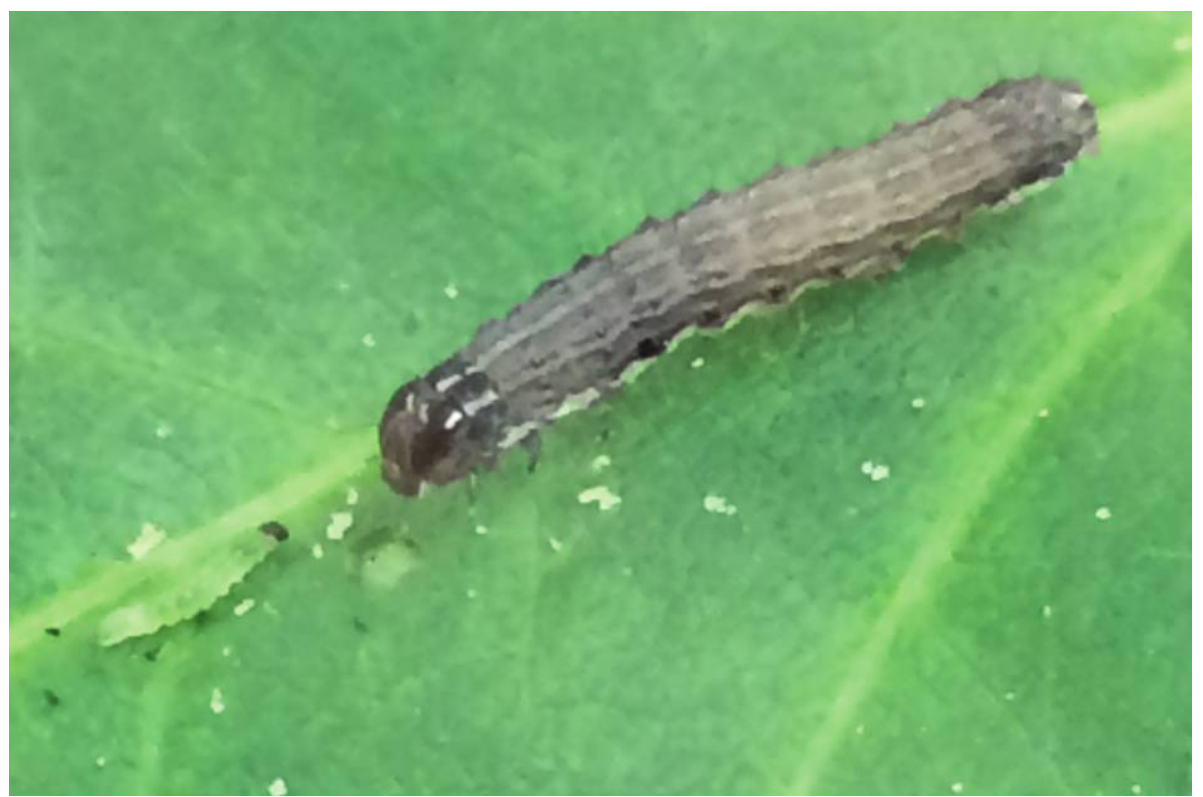

Figure 4: Two fall armyworm (Spodoptera frugiperda) caterpillars of the same age (within $24 \mathrm{~h}$ ). The caterpillar on the left was infected with the baculovirus Autographica californica multinucleate nucleopolyhedrovirus (AcMNPV) shortly after hatching and remained at that stage, while the uninfected larger caterpillar continued to grow and is in the fourth instar in this photo. The smaller, infected individual is a very inviting target for cannibalism by its much larger conspecific.

To understand how cannibalism may affect disease dynamics in such a stage-structured population, we constructed a model using a suite of differential equations based on an SEIR (susceptible, exposed, infectious, or recovered) model (Fuller et al. 2012; Elderd and Reilly 2014) modified for this cannibalistic insect host-parasite interaction:

$$
\begin{aligned}
& \frac{d S}{d t}=-\beta S P-f a S \sum_{i-1}^{m} E_{i}, \\
& \frac{d E_{1}}{d t}=\beta S P+f a S \sum_{i-1}^{m} E_{i}-m \delta E_{1}-a S E_{1}, \\
& \frac{d E_{i}}{d t}=m \delta E_{i-1}-m \delta E_{i}-a S E_{i,} i=2 \ldots m, \\
& \frac{d P}{d t}=m \delta E_{m}-\mu P .
\end{aligned}
$$

Here susceptible individuals, $S$, move into the first exposed stage, $E_{1}$, as determined by the transmission rate, $\beta$, and the amount of pathogen, $P$, in the system. Susceptible individuals can also become infected by attacking and consuming exposed individuals, $E_{i}$, of various stages $(1 \ldots m)$ given the attack rate, $a$, and the fraction of attacks, $f$, that result in an infection. Individuals leave the first exposed stage, $E_{1}$, either by moving to the next stage at rate $m \delta$ or by being consumed by a susceptible individual. Eventually, exposed individuals move through all $m$ stages and liquefy to become pathogen, $P$. The pathogen degrades over time due to UV sunlight exposure (Fuller et al. 2012) at rate $\mu$. There are no recovered individuals in the model, as the parasite is fatal once infected. The exposed and infected $\left(E_{i}\right)$ individuals move through a number of discrete stages during which the pathogen develops in the host. Almost all microparasites have some latent stage before peak infectiousness (Fraser et al. 2004); the length of the baculovirus latent stage is likely due to the dramatic liquefaction of host tissue necessary for transmission to occur (Fuller et al. 2012; Elderd 2013). The total time in the exposed stage is the sum of $m$ exponential distributions, which is a gamma distribution with a mean time in the exposed class of $1 / \delta$ and a variance of $\left(1 / m \delta^{2}\right)$. This model describes a single epidemic of baculovirus infection in a cohort of fall armyworms (Spodoptera frugiperda). Note, we assume only susceptible individuals are cannibals and all exposed individuals are equally infectious. The former holds if healthy individuals readily consume infected individuals. The latter is an assumption that can be relaxed but could be considered conservative.

Under this stage-structured framework (fig. 5), the cumulative fraction of individuals infected during a disease outbreak is always less than if a population was noncannibalistic at relatively high host densities (solid lines as compared to the dashed lines). At low host densities, cannibalism can slightly increases transmission of the disease but only when the cannibalistic transmission rate is very high (e.g., 90\% in fig. 5A). However, as host density increases, the disease reduction effects of cannibalism becomes stronger with increasing cannibalism rates. Interestingly, infec- 

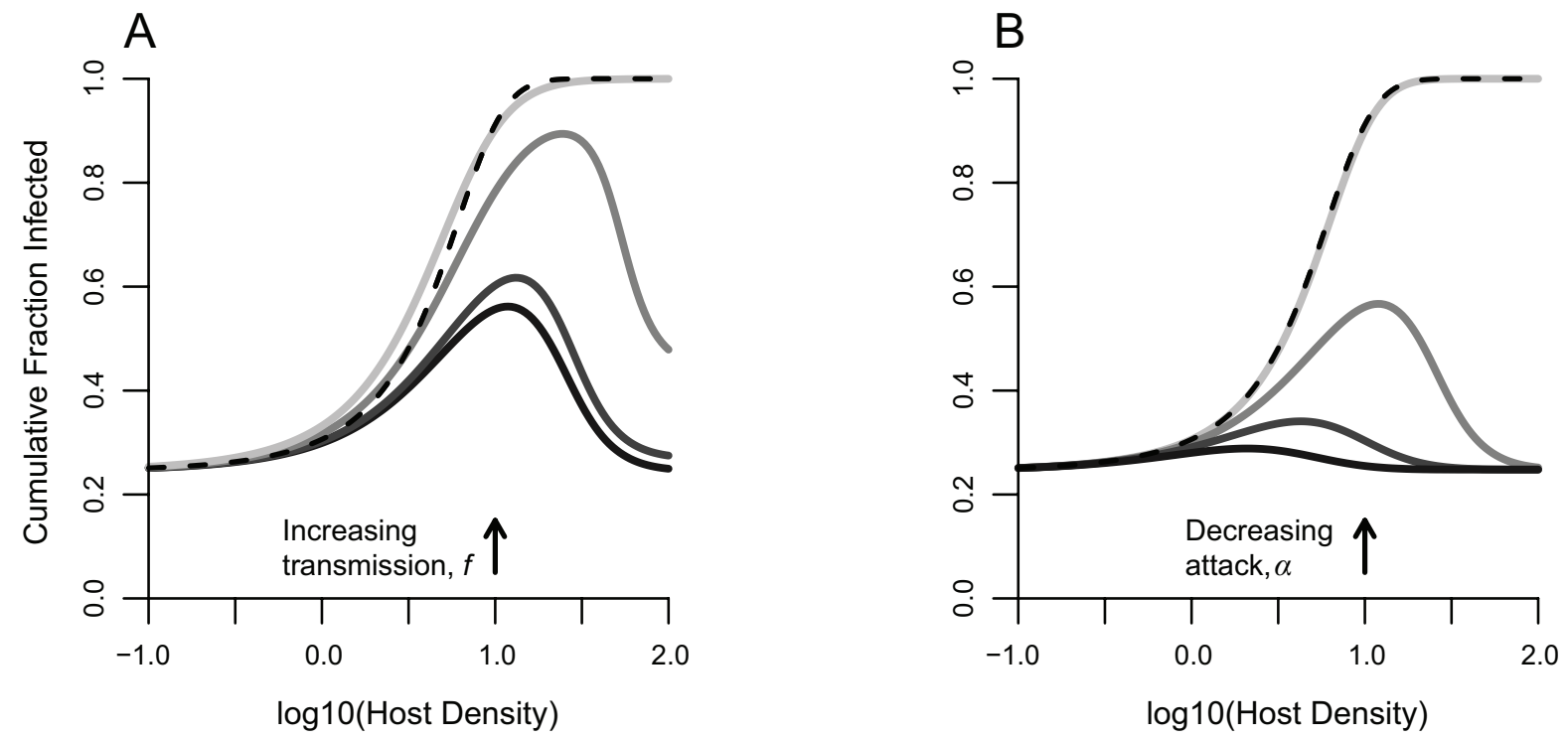

Figure 5: Effects of cannibalism on the cumulative fraction of individuals infected during a disease outbreak. Only infected individuals are cannibalized, and host pupation occurs after 30 days. Cannibalism rates of infected individuals are similar to what might be expected in lepidopteran host-pathogen systems where the host is cannibalistic, such as in fall armyworms (Spodoptera frugiperda). In both plots, the dashed line represents noncannibalistic populations. As transmission rate of the parasite through cannibalism increases, the dynamics become more similar to noncannibalistic populations (dark to light solid gray lines). $A$, The attack rate, $\alpha$, equals 0.02 ; the number of stages, $m$, equals $8 ; \delta$ equals $1 / 7.2$; and the cannibalistic transmission rate of the parasite increases from 0 (darkest gray solid line) to $0.1,0.5$, and 0.9 (lightest gray solid line). $B$, The cannibalistic transmission rate, $f$, equals 0.01 ; the number of stages $m$ equals 8 ; $\delta$ equals $1 / 7.2$; and the attack rate increases from 0.001 (lightest gray solid line) to $0.02,0.05$, and 0.1 (darkest gray solid line). In general, as the attack rate decreases, the overall fraction infected increases.

tion rates peak at medium host densities in cannibalistic populations and decline as host density increases, while infection rates simply increase to $100 \%$ in noncannibalistic populations (fig. 5). This difference in patterns of infection frequency between cannibalistic and noncannibalistic populations suggests that the nonlinear effects of cannibalism seen in equation (2) operate similarly here.

Our work with $S$. frugiperda shows that fourth-instar larvae consuming infected first-instar larvae are infected less than $10 \%$ of the time, suggesting that cannibalism could effectively reduce infection rates in real populations (fig. 5; B. G. Van Allen and B. D. Elderd, unpublished data). While the figure shows epizootic dynamics when larvae pupate after 30 days, which is reasonable for the fall armyworm, the dynamics change very little if the pupation time is either increased or decreased. In this system, cannibalism could have a greater effect toward reducing disease, as infected larger individuals remain cannibalistic for days. The infection progresses more slowly in these larger hosts, but they then produce far more viral particles when they liquefy, whether they acquire the disease from cannibalism or horizontal transmission (Valicente et al. 2013). By the time this happens, however, the rest of the uninfected cohort will have pupated and any virus produced will have to survive to infect a new cohort. Overall, stage-structured cannibalism and the higher proba- bility of smaller stages becoming infected and cannibalized in many systems could act to increase the impacts of the per capita and population-level effects of cannibalism on parasite transmission.

Through these three individual- and population-level mechanisms, cannibalism should tend to reduce the prevalence and/or fitness of parasites invading cannibalistic populations. One or more of these mechanisms most likely operates in virtually every cannibalistic population (table 1). The three models presented here are the first logical steps to examine the dynamics of cannibalism and disease spread, but they focus on simple (albeit common) scenarios. However, particular life histories and parasite strategies in the real world could potentially alter the relationship between cannibalism and disease. In the remainder of this article, we examine how widely different outcomes in the relationship between cannibalism and parasites are possible, first primarily from the perspective of variation in cannibals and then from the perspective of variation in parasites.

\section{Devil in the Details: A Cannibal's Perspective}

Cannibalism increases the chance of becoming infected when parasites capable of surviving the transmission from victim to cannibal occur in the population (i.e., fig. 3). This increased 


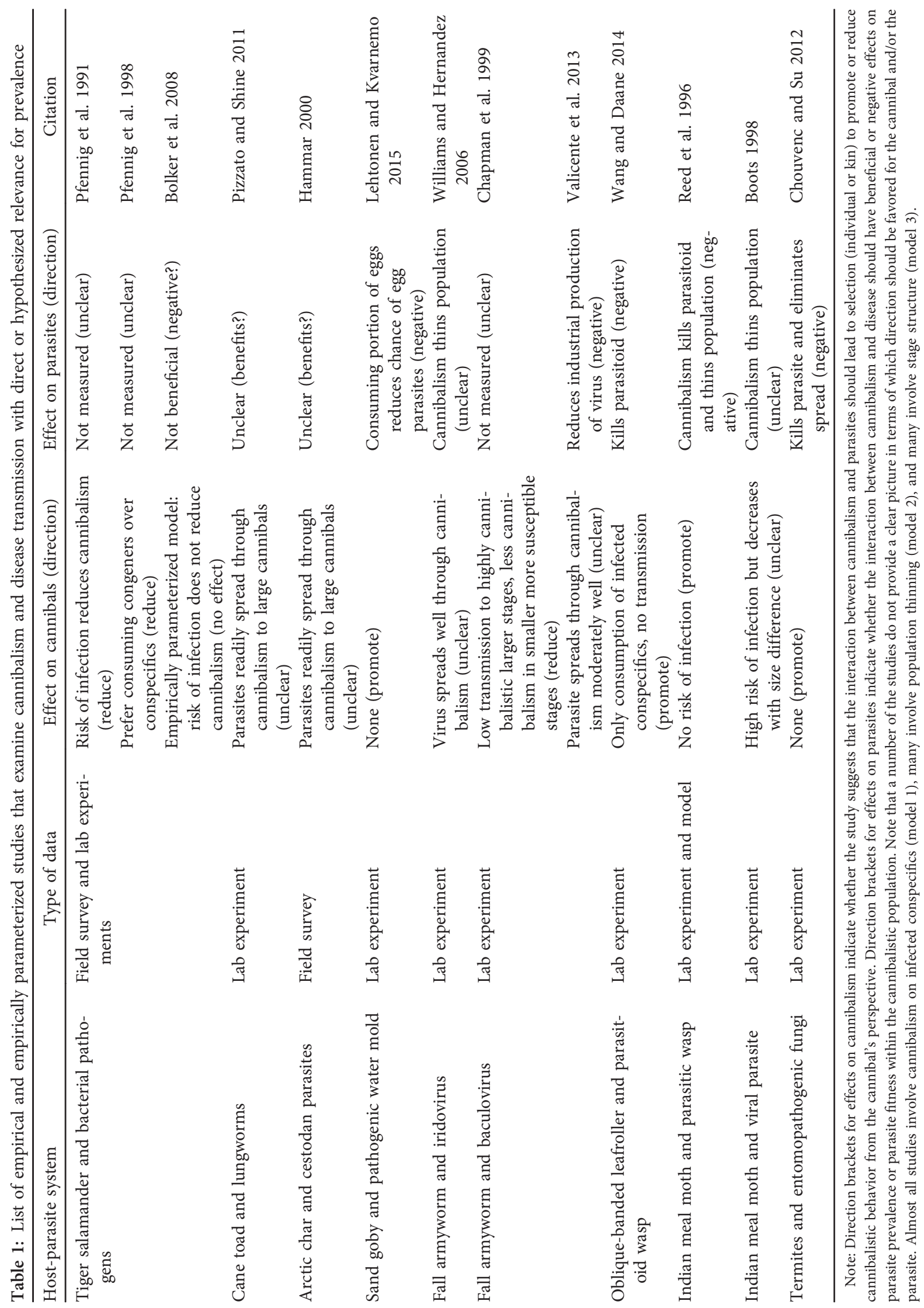

This content downloaded from 128.042.224.004 on September 18, 2017 09:25:56 AM 
risk relative to noncannibals would seem to select against the evolution of cannibalism that specifically targets infected individuals. Indeed, across a number of systems, there is evidence of a behavioral tendency to avoid infected conspecifics (Boots 1998; Pfennig et al. 1998; Crossland et al. 2011; Curtis 2014), though a tendency to cannibalize smaller and weaker conspecifics can directly oppose this (Boots 1998; Pizzato and Shine 2011). However, when there is no risk of infection for cannibals that consume infected individuals, it appears that targeted cannibalism of infected individuals can occur. For example, the targeted consumption of cannibalized conspecifics when risk to cannibals is nonexistent could help explain why some pathogens fail to control their hosts. The entomopathogenic fungus Metarhizium anisopliae has long been considered a promising candidate for biocontrol of subterranean termites. Yet, attempts to induce an epidemic of this pathogen in natural colonies have proved fruitless for decades (Chouvenc et al.2011). This could be at least partially driven by termite behavior: termites use burial and cannibalism of moribund individuals to prevent the transmission of fungal pathogens within their colonies (Rosengaus and Traniello 2001; Chouvenc and Su 2012; Meunier 2015). Importantly, M. anisopliae does not transmit through the gut and does not form disease-causing spores until after the host is dead, making cannibalism of moribund individuals nearly risk free (Rosengaus and Traniello 2001). Accounting for the coordinated cannibalism and burial of infected conspecifics, $75 \%$ of a termite population must simultaneously become infected with a lethal dose of the pathogen to cause an epidemic that will destroy the colony, which is highly unrealistic in structurally complex subterranean termite colonies (Chouvenc and Su 2012). Reports of caterpillars of lepidopteran species preferentially or only consuming conspecifics with either nontransmissible parasitoids (Reed et al. 1996; Wang and Daane 2014) or transmissible parasitoids (Boots 1998) suggests that this phenomenon is worthy of further examination. Ignoring the negative effects of cannibalism on parasites can be a costly mistake for those interested in using parasites for biocontrol (Chouvenc et al. 2011).

While examples of targeted cannibalism of infected conspecifics are rare in the literature, this behavior may be more common and simply overlooked, especially if cannibalism occurs before individuals become visibly sick (to the researcher). While caution is warranted in ascribing adaptive significance to a species' behavior, whether it avoids or targets infecteds (Poulin 1995), it seems that killing a parasite in a lethally infected host before it is able to further transmit can have clear selective benefits for an uninfected cannibal and its relatives. Future studies are needed to determine how frequent this behavior is in nature.

The relationship between individual risk of infection through cannibalism and the ability of cannibalism to reduce the prevalence of disease could be an important factor leading to variation in the rate of cannibalism between pop- ulations or species. Based on the models in this article and others, the correlations between individual risk of infection during cannibalism and the population-level frequency of cannibalism should often be negative (eqq. [2], [3]; fig. 3; Rudolf and Antonovics 2007). However, as the prevalence and transmission rate of a parasite increases, higher cannibalism rates are required to reduce parasite prevalence as transmission rate increases (i.e., fig. 2 and comparisons between panels in fig. 3). As a result, higher parasite transmission rates could select against increased cannibalism rates, until higher cannibalism rates reduce risk sufficiently to balance it against the associated benefits of cannibalism. Interestingly, this eco-evolutionary relationship between cannibalism rate, individual risk, and parasite transmission rate mirrors other work on cannibalism in dispersing populations (Rudolf et al. 2010). Species or populations that start with low cannibalism rates could be less likely to reach the point where cannibalism reduces disease and more likely to find risk of infection selecting against cannibalism. In any case, while low cannibalism rates and high pathogen prevalence may make cannibalism individually risky, it still would tend to reduce parasite prevalence and thus also be risky for the parasite (fig. 3; Rudolf and Antonovics 2007; Bolker et al. 2008).

Cannibalism as a way to specifically remove individuals to prevent disease spread could potentially also help explain the conundrum of filial cannibalism. Cannibalism is expected to be most common in animals when consuming close relatives (kin) is unlikely (Fox 1975; Polis 1981; Pfennig 1997; Rudolf et al. 2010). However, the practice of filial cannibalism, the consumption of eggs or juveniles by parents, is a widespread exception to this rule (Polis 1981; Smith and Reay 1991; Elgar and Crespi 1992; Richardson et al. 2010). By consuming weak or recently infected individuals, parents could protect their healthier offspring from later infection through eliminating current parasites and a reduction in clutch/litter density (Polis 1981; Hart 1990; Elgar and Crespi 1992; Richardson et al. 2010; Lehtonen and Kvarnemo 2015). This possibility ties well to recent work suggesting that the strength of kin selection varies with estimated future fitness (Dugas et al. 2016) and suggests that filial cannibalism could be another estimation of risk (including the risk of disease transmission) balanced against offspring potential (Lehtonen and Kvarnemo 2015). While additional explanations for filial cannibalism exist, such as maintaining balance between future costs of offspring rearing and resource levels (Fox 1975; Rohwer 1978; Polis 1981; Manica 2002; Klug and Bonsall 2007), the casting of filial cannibalism as a mechanism that reduces the prevalence of parasites is similar to a smallerscale version of the culling through cannibalism concept outlined above. This suggests that diseases may actually promote evolution of filial cannibalism, but this intriguing hypothesis remains to be tested (but see Lehtonen and Kvarnemo 2015). 
Across the diversity of hosts and pathogens, the effect of cannibalism on parasite transmission in the population could vary for a number of reasons. Diseases with a longer latent period before they become transmittable are more susceptible to removal through (even incidental) cannibalism, as they are also more likely to be removed through quarantine and other mechanisms that remove infected individuals from the wider population (Hart 1990; Fraser et al. 2004; Chouvenc and Su 2012; Wang and Daane 2014). If the parasite induces traits in infected individuals that make them less likely to be consumed, or if the parasite is transmitted through frequency dependence instead of density dependence (thereby removing a specific population size threshold for disease spread), the strength of the density-dependent effects of cannibalism are likely to be reduced (Potapov et al. 2012; Rudolf et al. 2012). Parasites can also increase the cannibalistic behavior of hosts, which can indirectly increase (i.e., by consuming susceptibles) or directly decrease (i.e., by consuming infecteds) the prevalence of the parasite, as well as decrease population density (Bunke et al. 2015). When a pathogen invades the community at a high rate, such as when a co-occurring population of a different species or an environmental source transmits the disease to the cannibalistic population, the ability of cannibalism to reduce parasite frequency could be overwhelmed and cannibalism could perhaps even increase the rate of parasite spread (Pfennig et al. 1991; Altizer et al. 2003, 2011; Rudolf and Antonovics 2005; McCallum 2012). In this spillover scenario, a disease could perhaps more easily select against cannibalism. However, even in this case, models suggest that the risk of disease through cannibalism must be both high and persistent (Bolker et al. 2008).

\section{Devil in the Details: A Parasite's Perspective}

While cannibalism of infected hosts can certainly be risky for susceptible cannibals, the parasite risks death with a best-case alternative of infecting a new host. Even if the parasite infects the new host, this will simply replace the old host with a new one in most situations (Rudolf and Antonovics 2007). However, there are a number of life-history strategies a pathogen can adopt to avoid or ameliorate a reduction in its prevalence/fitness in a cannibalistic population. Cannibalism most simply represents an increased mortality rate of infected and susceptible hosts. Theory predicts that increased host mortality will favor faster onset of transmission and higher transmission rates for the parasite, possibly resulting in higher virulence and, subsequently, faster death rates (Anderson and May 1982; Ewald 1983; Gandon et al. 2001). Decreasing the latent period and beginning to spread more quickly also gives cannibals less time to consume infected hosts. Surviving cannibalism by infecting the cannibal still usually results in fewer available hosts and forces the parasite to start over in the new host, delaying further trans- mission. While surviving cannibalism and increasing virulence may ameliorate a bad situation to insure continued infection of the host, these potential parasite responses will not directly result in cannibalism increasing the spread of disease.

Parasites infecting populations with stage-structured cannibalism can also avoid the negative effects of cannibalism by simply targeting the larger, cannibalistic individuals that are immune to cannibalism themselves (Rudolf et al. 2012). However, larger individuals may be less vulnerable to infection (Feng et al. 1985; Briggs and Godfray 1995; Boots 1998; Boff et al. 2000; Kelehear et al. 2009; Haislip et al. 2011; Harrison and Hoover 2012), and in the absence of cannibalism, competition among parasites typically imposes strong selection for a parasite to attack the younger stages of their hosts (Rudolf et al. 2012). Parasites that specialize in infecting the cannibalistic stage can escape the direct effects of cannibalism entirely but must be capable of selecting and infecting these individuals and are left with only those hosts who have made it through the earlier stage (Rudolf et al. 2012). However, when cannibals specialize on infected victims without regard to size, no stage may be safe. Whether cannibalism is rare or common in a population, it likely has negative effect on parasites.

Despite the generally negative impact of cannibalism on parasite spread and fitness, there are situations and systems where cannibalism could increase the spread of disease (Sadeh and Rosenheim 2016; Sadeh et al. 2016). First, the parasite must be capable of surviving cannibalistic encounters and transferring to the cannibal. Second, if this new host has higher survival, more resources, or perhaps has contact with more new susceptible hosts, then the parasite could find itself in a much better situation than in its original host. This situation can provide a clear benefit for the parasite (Pizzato and Shine 2011), especially when small hosts are both more susceptible to parasites and more likely to die in other ways before the parasite can reproduce (e.g., through predation or other parasite infections). As parasites are transferred to safe cannibalistic hosts through cannibalistic transmission and small/vulnerable individuals die off due to their higher rate of mortality, the overall rate of infection can increase by default even without further transmission (Hammar 2000; Pizzato and Shine 2011). Reaching these safe hosts also allows the parasite to reach the part of the population not regulated by cannibalism and escape its effects. This effect requires specific life histories and situations for both the parasite and host, but there is evidence that it occurs in multiple taxa (Hammar 2000; Pizzato and Shine 2011), and more work is needed to determine how often parasites can use cannibalism to their advantage this way.

In combination with certain alternative modes of transmission, cannibalism could act to increase parasite spread instead of decreasing it. In situations where a disease is 
transmitted vertically (from mother to offspring only), recent work indicates that cannibalism can indirectly increase disease prevalence by reducing the number of susceptibles while infected juveniles are added to the population (Sadeh and Rosenheim 2016). An example of this is likely found in the group of microsporidian pathogens that infect freshwater amphipods (MacNeil et al. 2003). Multiple sources assert that these pathogens are spread solely through cannibalism (MacNeil et al. 2003; Bunke et al. 2015). However, microsporidian parasites of freshwater amphipods are additionally vertically transmitted and alter population sex ratios (Bandi et al. 2001; Terry et al. 2004). Both factors would not be apparent in many experimental designs but could facilitate pathogen persistence in the population by themselves (Hurst 1993). Infected amphipods are also more cannibalistic, which could additionally increase the production of infected offspring without any further cannibalistic disease transmission (Bunke et al. 2015). In this way, cannibalism could work with vertically transmitted parasites to increase parasite spread (Sadeh and Rosenheim 2016).

Our review indicates that the relationship between cannibalism and disease could be a critical link in the spread of parasites in a wide range of animal populations. Yet this link has received surprisingly limited attention. The low recognition of the importance of cannibalism for reducing the prevalence of infectious disease likely stems from a number of direct and indirect biases on our measurements and perceptions. There has been a long-standing unjustified stigma against cannibalism implying that it should be rare in nature (Eibl-Eibelsfelt 1961). As a consequence, cannibalism is still highly understudied, especially as related to its interaction with parasitism, despite its high frequency across the animal kingdom. Cannibalism is most likely noticed in species with many offspring and relatively fast life histories, such as invertebrates, fish, and many reptile and amphibian species (Polis and Myers 1985; Smith and Reay 1991; Ibáñez and Keyl 2010; Richardson et al. 2010), and since diseases are much less understood or studied in invertebrates and other smaller animals than in large vertebrates, the empirical evidence linking cannibalism to disease is also less well known. Cannibalism is generally less frequent in the largest vertebrates as well, barring certain social species (Fox 1975; Polis 1981; Fernandez-Jalvo et al. 1996; Rudolf and Antonovics 2007), leading to the situation where cannibalism is not common enough to significantly reduce disease, yet parasites could still perhaps spread through it (Forbes 2000; Pozio 2013). Additionally, noting the presence of disease is perhaps much more compelling than noting the absence of disease in an organism or population. As a result, the attention paid to exceptional systems where cannibalism appears to readily transmit parasites and spread disease could overshadow other systems in which cannibalism reduces disease spread or has little effect on it. In addition to the cultural taboo of cannibalism, these factors likely play a major role in slowing our recognition of the impacts of cannibalism on infectious disease.

\section{Future Directions}

The progress synthesized in this review reveals the importance of eco-evolutionary feedbacks between cannibalism and infectious diseases and suggests a range of open and exciting avenues for future research. We show a number of mechanisms through which cannibalism can reduce parasite prevalence and spread, while in many cases also reducing the negative selective effects of parasites on cannibals. We additionally highlight the mechanisms through which cannibalism sometimes could enhance the spread of disease (Sadeh and Rosenheim 2016; Sadeh et al. 2016) and rarer cases where it appears that cannibalistic behavior could have arisen to combat or avoid parasites (Chouvenc and Su 2012; Lehtonen and Kvarnemo 2015; table 1).

Empirical examinations of the relationship between cannibalism and pathogen prevalence in natural populations, however, are few and scattered (Pfennig et al. 1991; Reed et al. 1996; Chouvenc and Su 2012; Lehtonen and Kvarnemo 2015), and more empirical work in natural or laboratory settings is needed. While theory suggests that cannibalism should reduce the prevalence of parasites and their ability to invade the population, this is largely untested. Similarly, it is unknown which of the different mechanisms (such as per capita vs. population density) are most important, and whether there are systematic differences across taxa groups, life histories, or environments.

The evolutionary consequences of cannibalism for parasite life history are largely unstudied (Rudolf et al. 2012; table 1). In this review, we outline predictions from general epidemic theory that could be tested along gradients of cannibalistic behavior in a suitable system. Whether cannibalism could select for earlier onset of transmission, increased noncannibalistic transmission, and/or increased cannibalistic transmission separately or simultaneously-and how this might vary between different life histories and systems - is an open question. Changes to parasite life history and prevalence could feed back to alter cannibalistic behavior as well. Evolutionary theory predicts that cannibalism and disease should have an antagonistic relationship, with the prevalence of each selecting against the other (Pfennig et al. 1991, 1998; Bolker et al. 2008), though which process limits the other more strongly and how their interactions play out in terms of both traits (evolution) and through host and pathogen dynamics (ecology) remain open questions.

The effects and importance of the interactions between cannibalism and disease in different environments, life histories, and systems will certainly vary, and a number of approaches will be needed to understand this important connection between parasites and the life history of their 
hosts. More natural history data on parasite prevalence and cannibalism rates is essential to determining the relationship between cannibalism and disease across taxa (Tewksbury et al. 2014). Combined natural history, experiments, and models have provided much of the work on cannibalism and disease thus far (Pfennig et al. 1991; Reed et al. 1996; Boots 1998; Bolker et al. 2008; Pizzato and Shine 2011; Lehtonen and Kvarnemo 2015; Sadeh and Rosenheim 2016; Sadeh et al. 2016), but much more work across taxa is needed to identify general patterns. Once more empirical investigations of these issues have been completed, a meta-analysis of their results and the prevalence of cannibalistic behavior and parasitism rates for populations or species could be used along with theoretical work to provide evidence to support or weaken the arguments outlined in this article and determine any potential phylogenetic patterns. Such a meta-analysis would provide much needed direction for future work in understanding the ecological and evolutionary relationships between cannibalism and parasite transmission.

\section{Conclusion}

In this review, we have shown that across taxa, systems, and life histories, cannibalism is often likely to decrease the prevalence or spread of parasites. From the perspective of a population or an epidemiologist then, cannibalism should reduce the spread of disease. From an individual perspective, we have shown that the risk of infection is often very low, but in certain situations it could be substantial (Pfennig et al. 1998; Rudolf and Antonovics 2007). As a result, cannibalism and parasites may find themselves in an antagonistic situation, both inflicting costs on each other (Pfennig et al. 1998; Bolker et al. 2008). However, in many cases, it seems that cannibalism should reduce disease prevalence and thereby decrease the negative selective effect of parasites on cannibalism, and not vice versa (Rudolf and Antonovics 2007; Bolker et al. 2008). This reduction in the effects of parasites on cannibals could then allow the direct positive effects of cannibalism (resources, reduced competition) to become more heavily weighted, balanced only against the other classic negative effects (injury and killing kin; Polis 1981; Rudolf et al. 2010). In sum, we propose that the multiple mechanisms by which cannibalism reduces the risk of disease tend to reduce the negative effects of disease on cannibalism beyond what is usually appreciated. The focus of much work on cannibalism posits that cannibalism may transmit disease and that the associated negative effects of disease transmission may select against cannibalism. As we have shown, this is in conflict with recent theoretical and empirical work. More research is needed to clarify the relationship between cannibalism and disease, and what governs variation in this relationship between systems. Un- til this is done, we provided some building blocks that can be used to demonstrate that the relationship between cannibalism and disease is complex but can often involve more costs for the parasite than for the hosts, thus reducing the prevalence of disease.

\section{Acknowledgments}

This work was funded by National Science Foundation (NSF) grant 1316334 as part of the joint NSF-National Institutes of Health-USDA Ecology and Evolution of Infectious Diseases program. We thank T. Lyles for insightful commentary on a draft of the manuscript.

\section{Literature Cited}

Altizer, S., R. Bartel, and B. A. Han. 2011. Animal migration and infectious disease risk. Science 331:296-302.

Altizer, S., D. Harvell, and E. Friedle. 2003. Rapid evolutionary dynamics and disease threats to biodiversity. Trends in Ecology and Evolution 18:589-596.

Anderson, R. M., and R. M. May. 1980. Infectious diseases and population cycles of forest insects. Science 210:658-661.

. 1982. Coevolution of hosts and parasites. Parasitology $85: 411-426$

Arcadi, A. C., and R. W. Wrangham. 1999. Infanticide in chimpanzees: review of cases and a new within-group observation from the Kanyawara study group in Kibale National Park. Primates 40:337-351.

Bandi, C., A. M. Dunn, G. D. Hurst, and T. Rigaud. 2001. Inherited microorganisms, sex-specific virulence and reproductive parasitism. Trends in Parasitology 17:88-94.

Bertram, B. C. 1975. Social factors influencing reproduction in wild lions. Journal of Zoology 177:463-482.

Boff, M. I. C., G. L. Wiegers, and P. H. Smits. 2000. Influences of host size and host species on the infectivity and development of Heterorhabditis megidis (strain NLH-E87. 3). BioControl 45:469-482.

Bolker, B. M., F. De Castro, A. Storfer, S. Mech, E. Harvey, and J. P. Collins. 2008. Disease as a selective force precluding widespread cannibalism: a case study of an iridovirus of tiger salamanders, Ambystoma tigrinum. Evolutionary Ecology Research 10:105-128.

Boots, M. 1998. Cannibalism and the stage-dependent transmission of a viral pathogen of the Indian meal moth, Plodia interpunctella. Ecological Entomology 23:118-122.

Briggs, C. J., and H. C. J. Godfray. 1995. The dynamics of insectpathogen interactions in stage-structured populations. American Naturalist 145:855-887.

Brooks-Pollock, E., G. O. Roberts, and M. J. Keeling. 2014. A dynamic model of bovine tuberculosis spread and control in Great Britain. Nature 511:228-231.

Bunke, M., M. E. Alexander, J. T. A. Dick, M. J. Hatcher, R. Paterson, and A. M. Dunn. 2015. Eaten alive: cannibalism is enhanced by parasites. Roval Society Open Science 2:140369-140369.

Chapman, J. W., T. Williams, A. Escribano, P. Caballero, R. D. Cave, and D. Goulson. 1999. Age-related cannibalism and horizontal transmission of a nuclear polyhedrosis virus in larval Spodoptera frugiperda. Ecological Entomology 24:268-275.

Chouvenc, T., and N.-Y. Su. 2012. When subterranean termites challenge the rules of fungal epizootics. PLoS ONE 7:e34484. 
Chouvenc, T., N.-Y. Su, and J. Kenneth Grace. 2011. Fifty years of attempted biological control of termites: analysis of a failure. Biological Control 59:69-82.

Claessen, D., A. M. De Roos, and L. Persson. 2004. Population dynamic theory of size-dependent cannibalism. Proceedings of the Roval Society B 271:333-340.

Collinge, J. 2001. Prion diseases of humans and animals: their causes and molecular basis. Annual Review of Neuroscience 24:519-550.

Collinge, J., J. Whitfield, E. McKintosh, J. Beck, S. Mead, D. J. Thomas, and M. P. Alpers. 2006. Kuru in the 21st century: an acquired human prion disease with very long incubation periods. Lancet 367: 2068-2074.

Crossland, M. R., M. N. Hearnden, L. Pizzatto, R. A. Alford, and R. Shine. 2011. Why be a cannibal? the benefits to cane toad, Rhinella marina [ = Bufo marinus], tadpoles of consuming conspecific eggs. Animal Behaviour 82:775-782.

Curtis, V. A. 2014. Infection-avoidance behaviour in humans and other animals. Trends in Immunology 35:457-464.

Dawkins, R. 1976. The selfish gene: 30th anniversary edition. Oxford University Press, Oxford.

Dugas, M. B., L. McCormack, A. Gadau, R. A. Martin, L. J. Harmon, and Y. Michalakis. 2016. Choosy cannibals preferentially consume siblings with relatively low fitness prospects. American Naturalist 188:124-131.

Eibl-Eibelsfelt, I. 1961. The fighting behavior of animals. Scientific American 205:112-123.

Elderd, B. D. 2013. Developing models of disease transmission: insights from ecological studies of insects and their baculoviruses. PLoS Pathogens 9:e1003372.

Elderd, B. D., J. Dushoff, and G. Dwyer. 2008. Host-pathogen interactions, insect outbreaks, and natural selection for disease resistance. American Naturalist 172:829-842.

Elderd, B. D., and J. R. Reilly. 2014. Warmer temperatures increase disease transmission and outbreak intensity in a host-pathogen system. Journal of Animal Ecology 83:838-849.

Elgar, M. A., and B. J. Crespi. 1992. Cannibalism: ecology and evolution among diverse taxa. Oxford University Press, Oxford.

Ewald, P. W. 1983. Host-parasite relations, vectors, and the evolution of disease severity. Annual Review of Ecology and Systematics 14:465-485.

Feng, Z., R. I. Carruthers, D. W. Roberts, and D. S. Robson. 1985 Age-specific dose-mortality effects of Beaiveria bassaiana (Dueteromycotina: Hyphomycetes) on the European Corn Borer, Ostrinia nubalis (Lepidoptera: Pyralidae). Invertebrate Pathology 46:259-264.

Fernandez-Jalvo, Y., J. C. Diez, J. M. B. de Castro, E. Carbonell, and J. L. Arsuaga. 1996. Evidence of early cannibalism. Science 271:277-278

Forbes, L. B. 2000. The occurrence and ecology of Trichinella in marine mammals. Veterinary Parasitology 93:321-334.

Fox, L. R. 1975. Cannibalism in natural populations. Annual Review of Ecology and Systematics 6:87-106

Fraser, C., S. Riley, R. M. Anderson, and N. M. Ferguson. 2004. Factors that make an infectious disease outbreak controllable. Proceedings of the National Academy of Sciences of the USA 101:6146-6151.

Fuller, E., B. D. Elderd, and G. Dwyer. 2012. Pathogen persistence in the environment and insect-baculovirus interactions: diseasedensity thresholds, epidemic burnout, and insect outbreaks. American Naturalist 179:E70-E96.

Gandon, S., V. A. Jansen, and M. Van Baalen. 2001. Host life history and the evolution of parasite virulence. Evolution 55:1056-1062.
Haislip, N. A., M. J. Gray, J. T. Hoverman, and D. L. Miller. 2011 Development and disease: how susceptibility to an emerging pathogen changes through anuran development. PLoS ONE 6:e22307.

Hammar, J. 2000. Cannibals and parasites: conflicting regulators of bimodality in high latitude Arctic char, Salvelinus alpinus. Oikos 88:33-47.

Harrison, R., and K. Hoover. 2012. Baculoviruses and other occluded insect viruses. Pages 73-131 in F. E. Vega and H. K. Kaya, eds. Insect pathology. Elsevier, London.

Hart, B. L. 1990. Behavioral adaptations to pathogens and parasites: five strategies. Neuroscience and Biobehavioral Reviews 14:273-294.

Hatcher, M. J., J. T. A. Dick, and A. M. Dunn. 2014. Parasites that change predator or prey behaviour can have keystone effects on community composition. Biology Letters, http://dx.doi.org/10.1098 /rsbl.2013.0879.

Hurst, L. D. 1993. The incidences: mechanisms and evolution of cytoplasmic sex ratio distorters in animals. Biological Reviews 68:121-194.

Ibáñez, C. M., and F. Keyl. 2010. Cannibalism in cephalopods. $\underline{\text { Re- }}$ views in Fish Biology and Fisheries 20:123-136.

Johnson, P. T., A. Dobson, K. D. Lafferty, D. J. Marcogliese, J. Memmott, S. A. Orlofske, R. Poulin, and D. W. Thieltges. 2010. When parasites become prey: ecological and epidemiological significance of eating parasites. Trends in Ecology and Evolution 25:362-371.

Kelehear, C., J. K. Webb, and R. Shine. 2009. Rhabdias pseudosphaerocephala infection in Bufo marinus: lung nematodes reduce viability of metamorph cane toads. Parasitology 136:919.

Klitzman, R. L., M. P. Alpers, and D. C. Gajdusek. 1984. The natural incubation period of Kuru and the episodes of transmission in three clusters of patients. Neuroepidemiology 3:3-20.

Klug, H., and M. B. Bonsall. 2007. When to care for, abandon, or eat your offspring: the evolution of parental care and filial cannibalism. American Naturalist 170:886-901.

Kuris, A. M. 1974. Trophic interactions: similarity of parasitic castrators to parasitoids. Quarterly Review of Biology 49:129-148.

Lafferty, K. D. 1999. The evolution of trophic transmission. Parasitology Todav 15:111-115.

Lehtonen, T. K., and C. Kvarnemo. 2015. Infections may select for filial cannibalism by impacting egg survival in interactions with water salinity and egg density. Oecologia (Berlin) 178:673-683.

Lindenbaum, S. 1979. Kuru sorcery: disease and danger in the New Guinea Highlands. Routledge, Abingdon-on-Thames, Oxfordshire.

MacNeil, C., J. T. A. Dick, M. J. Hatcher, N. J. Fielding, K. D. Hume, and A. M. Dunn. 2003. Parasite transmission and cannibalism in an amphipod (Crustacea). International Journal for Parasitology 33:795-798.

Manica, A. 2002. Filial cannibalism in teleost fish. Biological Reviews of the Cambridge Philosophical Society 77:261-277.

Matuschka, F.-R., and B. Bannert. 1989. Recognition of cyclic transmission of Sarcocystis stehlinii n. sp. in the Gran Canarian giant lizard. Journal of Parasitology 75:383.

McCallum, H. 2012. Disease and the dynamics of extinction. Philosophical Transactions of the Roval Society B 367:2828-2839.

McCallum, H., N. Barlow, and J. Hone. 2001. How should pathogen transmission be modelled? Trends in Ecology and Evolution 16:295-300.

McCallum, H., M. Jones, C. Hawkins, R. Hamede, S. Lachish, D. L. Sinn, N. Beeton, and B. Lazenby. 2009. Transmission dynamics of Tasmanian devil facial tumor disease may lead to disease-induced extinction. Ecology 90:3379-3392.

Meunier, J. 2015. Social immunity and the evolution of group living in insects. Philosophical Transactions of the Royal Society B, http:// dx.doi.org/10.1098/rstb.2014.0102. 
Miller, T. E. X., and V. H. W. Rudolf. 2011. Thinking inside the box: community-level consequences of stage-structured populations. Trends in Ecology and Evolution 26:457-466.

Mitani, J. C., D. P. Watts, and M. N. Muller. 2002. Recent developments in the study of wild chimpanzee behavior. Evolutionary Anthropology 11:9-25.

Moksnes, P.-O. 2004. Self-regulating mechanisms in cannibalistic populations of juvenile shore crabs Carcinus maenas. Ecology 85:1343-1354.

Ohlberger, J., Ø. Langangen, N. C. Stenseth, and L. A. Vøllestad. 2012. Community-level consequences of cannibalism. American Naturalist 180:791-801.

Park, E. J., C.-M. Yin, and J. P. Burand. 1996. Baculovirus replication alters hormone-regulated host development. Lournal of General Virology 77:547-554.

Pfennig, D. W. 1997. Kinship and cannibalism. Bioscience 47:667-675.

. 2000. Effect of predator-prey phylogenetic similarity on the fitness consequences of predation: a trade-off between nutrition and disease? American Naturalist 155:335-345.

Pfennig, D. W., S. G. Ho, and E. A. Hoffman. 1998. Pathogen transmission as a selective force against cannibalism. Animal Behaviour 55:1255-1261.

Pfennig, D. W., M. L. G. Loeb, and J. P. Collins. 1991. Pathogens as a factor limiting the spread of cannibalism in tiger salamanders. Oecologia (Berlin) 88:161-166.

Pizzato, L., and R. Shine. 2011. You are what you eat: parasite transfer in cannibalistic cane toads. Hepetologica 67:118-123.

Polis, G. A. 1981. The evolution and dynamics of intraspecific predation. Annual Review of Ecology and Systematics 12:225-251.

Polis, G. A., and C. A. Myers. 1985. A survey of intraspecific predation among reptiles and amphibians. Iournal of Herpetology 19:99.

Potapov, A., E. Merrill, and M. A. Lewis. 2012. Wildlife disease elimination and density dependence. Proceedings of the Roval Society B 279:3139-3145.

Poulin, R. 1995. "Adaptive" changes in the behaviour of parasitized animals: a critical review. International Journal for Parasitology 25:1371-1383.

Pozio, E. 2013. The opportunistic nature of Trichinella —exploitation of new geographies and habitats. Veterinary Parasitology 194:128-132.

Rausch, R. A. 1967. Some aspects of the population ecology of wolves, Alaska. American Zoologist 7:253-265.

Reed, D. J., M. Begon, and D. J. Thompson. 1996. Differential cannibalism and population dynamics in a host-parasitoid system. Oecologia (Berlin) 105:189-193.

Richardson, M. L., R. F. Mitchell, P. F. Reagel, and L. M. Hanks. 2010. Causes and consequences of cannibalism in noncarnivorous insects. Annual Review of Entomology 55:39-53.

Rohwer, S. 1978. Parent cannibalism of offspring and egg raiding as a courtship strategy. American Naturalist 112:429-440.

Rosengaus, R., and J. Traniello. 2001. Disease susceptibility and the adaptive nature of colony demography in the dampwood termite Zootermopsis angusticollis. Behavioral Ecology and Sociobiology 50:546-556.

Rudolf, V. H. 2008. Impact of cannibalism on predator-prey dynamics: size-structured interactions and apparent mutualism. Ecology 89:1650-1660.

Rudolf, V. H., and J. Antonovics. 2007. Disease transmission by cannibalism: rare event or common occurrence? Proceedings of the Roval Societv B 274:1205-1210.
Rudolf, V. H., I. Sorrell, and A. B. Pederson. 2012. Revenge of the host: cannibalism, ontogenetic niche shifts, and the evolution of life-history strategies in host-parasitoid systems. Evolutionary Ecology Research 14:31-49.

Rudolf, V. H. W., and J. Antonovics. 2005. Species coexistence and pathogens with frequency-dependent transmission. American Naturalist 166:112-118.

Rudolf, V. H. W., M. Kamo, and M. Boots. 2010. Cannibals in space: the coevolution of cannibalism and dispersal in spatially structured populations. American Naturalist 175:513-524.

Sadeh, A., T. D. Northfield, and J. A. Rosenheim. 2016. The epidemiology and evolution of parasite transmission through cannibalism. Ecology 97:2003-2011.

Sadeh, A., and J. A. Rosenheim. 2016. Cannibalism amplifies the spread of vertically-transmitted pathogens. Ecology 97:1994-2002.

Sait, S. M., M. Begon, and D. J. Thompson. 1994. The effects of a sublethal baculovirus infection in the Indian meal moth, Plodia interpunctella. Journal of Animal Ecology 63:541.

Smith, C., and P. Reay. 1991. Cannibalism in teleost fish. Reviews in Fish Biology and Fisheries 1:41-64.

Stenseth, N. C. 1985. On the evolution of cannibalism. Journal of Theoretical Biology 115:161-177.

Terry, R. S., J. E. Smith, R. G. Sharpe, T. Rigaud, D. T. J. Littlewood, J. E. Ironside, D. Rollinson, et al. 2004. Widespread vertical transmission and associated host sex-ratio distortion within the eukaryotic phylum Microspora. Proceedings of the Roval Society B 271:1783-1789.

Tewksbury, J. J., J. G. T. Anderson, J. D. Bakker, T. J. Billo, P. W. Dunwiddie, M. J. Groom, S. E. Hampton, et al. 2014. Natural history's place in science and society. BioScience 64:300-310.

Valicente, F. H., E. S. Tuelher, R. C. Pena, R. Andreazza, and M. R. F. Guimarães. 2013. Cannibalism and virus production in Spodoptera frugiperda (J.E. Smith) (Lepidoptera: Noctuidae) larvae fed with two leaf substrates inoculated with baculovirus Spodoptera. Neotropical Entomology 42:191-199.

Van Allen, B. G., and V. H. W. Rudolf. 2013. Ghosts of habitats past: environmental carry-over effects drive population dynamics in novel habitat. American Naturalist 181:596-608.

Vijendravarma, R. K., S. Narasimha, and T. J. Kawecki. 2013. Predatory cannibalism in Drosophila melanogaster larvae. Nature Communications 4:1789.

Wagner, J. D., and D. H. Wise. 1996. Cannibalism regulates densities of young wolf spiders: evidence from field and laboratory experiments. Ecology 77:639-652.

Wang, X.-G., and K. M. Daane. 2014. Cannibalism of parasitoidattacked conspecifics in a non-carnivorous caterpillar. Entomologia Experimentalis et Applicata 151:112-121.

Williams, T., and O. Hernández. 2006. Costs of cannibalism in the presence of an iridovirus pathogen of Spodoptera frugiperda. Ecological Entomology 31:106-113.

Wilson, M. L., W. R. Wallauer, and A. E. Pusey. 2004. New cases of intergroup violence among chimpanzees in Gombe National Park, Tanzania. International Journal of Primatology 25:523-549.

Wissinger, S. A., W. S. Brown, and J. E. Jannot. 2003. Caddisfly life histories along permanence gradients in high-altitude wetlands in Colorado (USA). Freshwater Biology 48:255-270.

Editor: Yannis Michalakis 